\title{
Stability and water control of nitrogen foam in bulk phase and porous media
}

\author{
Zhao Renbao $^{1,2 *}$, Hou Yongli ${ }^{1,2}$, Ke Wenqi ${ }^{1,2}$ and Yue Xiang'an ${ }^{1,2}$ \\ ${ }^{1}$ Key Laboratory of Petroleum Engineering, Ministry of Education, China University of Petroleum, Beijing 102249, China \\ ${ }^{2}$ Research Center of Enhanced Oil Recovery, China University of Petroleum, Beijing 102249, China
}

\begin{abstract}
Foam is widely used in the petroleum industry thanks to its unique properties and performance. Its application to water control in oil wells has received more and more attention. The stability of nitrogen foam was investigated in pressurized equipment and sand pack. The result indicated that with increasing pressure $(0-2 \mathrm{MPa})$ the stability of foam in the pressurized equipment increased linearly. Increased nitrogen injection pressure caused better dispersion of nitrogen-foaming solution in porous media. The initial residual resistance factor to gas was an exponential function of injection pressure, but the residual resistance factor (to gas and liquid) decreased exponentially with time. The halflife of foam in porous media (expressed in residual resistance factor) was much longer than that in bulk phase (expressed in foam height). Pore model analysis indicated that good dispersion in porous media and the presence of thick liquid film during dispersion were the main reasons for high stability. Field test indicated that effective residence of foam in the formation not only resulted in much better heat insulation, but also improved steam stimulation by enhancing steam heating.
\end{abstract}

Key words: Enhanced oil recovery, foam, porous media, permeability, residual resistance factor, residence time

\section{Introduction}

Most oil fields in China have matured with a medium to high water cut. Increasing water production has become a bottleneck restricting oil field development. Injection of gas as a means of enhanced oil recovery has attracted more and more attention (Greaves, 2004). Much progress has been made both in gas injection equipment and technology, and a great variety of reservoirs are using gas injection, such as strongly water sensitive reservoirs and ones with limited access to water supply (offshore reservoirs). Gas injection is the first choice for supplementing formation energy and extending oil field production. Early studies showed that foam can reduce gas mobility and control the pores with different permeability values (Bond and Holbrook, 1958; Bernard and Holm, 1964; Zhu et al, 2004), enhancing sweep efficiency of subsequent displacement fluids (Raza, 1970). Researchers (Rossen, 1990; Bergeron et al, 1993; Kovscek et al, 1997) studied the factors influencing foam production and its stability in porous media. These factors include pressure gradient, permeability, pore-throat size, pore length, gas/water ratio, oil, and pseudoemulsion film stability.

There are many measurable parameters describing the foam in bulk or in a beaker, such as liquid holdup (Ma, 1994), half-life and foam height (Iglesias et al, 1995), foam

*Corresponding author. email: zhaorenbao@vip.sina.com Received September 8, 2008 size distribution, and disjoining pressure (Jiang et al, 2000). In previous studies, optical measurement ( $\mathrm{Li}$ et al, 2007), micro-interferometry (Müller and Rheinländer, 1996), gamma ray (Deshpande and Barigou, 2001), electrical conductivity (Barigou et al, 2001), NMR (Kose, 1996), Langmuir-Blodgett film balance (Jiang et al, 2000), and Fourier transform infrared spectrometry (Cohen et al, 1997) were used.

Fewer methods are available for the study of foam in porous media, and only qualitative or statistical average results are generally obtained. The parameters described in the studies include pressure gradient and gas/liquid ratio, and the latter involves a greater error. Besides, foam, as a thermodynamically unstable system, changes with time and is very sensitive to the change in environment. The physical models used include one-dimensional model (Rossen, 2000), plate model (Kovscek et al, 2007), and sand-packed model (Holm, 1968). The study of Rossen showed that snap-off repeatedly occurred when gas flowed continuously from a wide pore space to the narrow pore throat. Kovscek proved that repeated snap-off of gas bubbles at pore throats is relevant to steady-state flow through porous media. Importantly, snap-off is shown to be the dominant mechanism for generation of a discontinuous gas phase within this porous medium. Holm observed the formation and breakage of liquid film in a $0.25 \mathrm{~mm}$ sand pack and presented a "breakage and rebirth" model. However, it is difficult to determine whether the system reaches the true stable state in such a setup.

The above description of foam appearance cannot explain 
its formation and breakdown in porous media, and many laboratory studies on the formation and distribution of foam liquid film are far from useful in guiding field application.

Because the gas-liquid interface in non-transparent porous cores cannot be directly determined, an alternative is to measure the flow resistivity and effective life of foam. The two critical indicators are not only related to the distribution of gas/liquid, but also to that of pore throats. Pore throat description is very complicated and its calculation is delivered only on the millimeter scale at present, which is much smaller than the reservoir reality. Therefore, the description of foam is generally by indirect measurement and numerical simulation.

Laboratory evaluation of foam generally uses pressure gradient, pressure fluctuation, resistance factor, and sweep efficiency to evaluate foam performance. In field application, extension of gas breakthrough time and increase of gas injection pressure are used to enhance its sweep efficiency and water/gas blockage effect. In addition to the above parameters, especially for the reservoir with active edge water or bottom water, the authors recommend using the residence time of the foam in the reservoir as an important indicator of evaluating its blockage or water-control effect, and to characterize its residence capability by using the change of residual resistance factor with time.

\section{Experimental}

\subsection{Equipment and materials}

A self-designed pressure-tight Plexiglas apparatus (shown in Fig. 1) was used to determine the stability of foam in bulk phase when the pressure was higher than atmospheric pressure. The equipment consisted of two parts: an upper part for pressure measurement connected with a pressure gauge; and a lower part of pressure-tight graduated cylinder made of Plexiglas tubes $(1.5 \mathrm{~cm}$ thick, $2 \mathrm{~cm}$ inside diameter, 60 $\mathrm{ml}$ volume). The equipment was pressure tested before use. Water was injected to build up pressure to $6 \mathrm{MPa}$, and stayed for 24 hours without leakage.

The core flooding system made by Jiangsu Hai'an Instrument Factory was used to determine permeability of sand pack to gas and water and evaluate foam stability in porous media. A schematic diagram is shown in Fig. 2. A Ross-Miles foam tester made by Beijing Glass Instrument Factory was used to determine the half-life of foam under atmospheric pressure.

The surfactant used in experiments was dodecyloxy tertiary amine, $\mathrm{CTAB}$, and $\mathrm{OP}-10 \mathrm{NaCl}, \mathrm{KCl}, \mathrm{CaCl}_{2}$, and $\mathrm{NH}_{4} \mathrm{NO}_{2}$ used were all commercial, analytical reagents. Outcrop sand, taken from Qinhuangdao, China, was crushed and screened through 160/100-mesh size sieves. The screened 100-160 mesh sands were washed with water and then dried at $80{ }^{\circ} \mathrm{C}$ for $24 \mathrm{~h}$ before use.

Simulated formation water was $0.2 \mathrm{wt} \% \mathrm{NaCl}$ solution, with total salinity of $2,000 \mathrm{mg} / \mathrm{L}$. Foaming solution was prepared by mixing $\mathrm{CTAB}$ and OP-10 in water, such that the solution contained about $0.15 \mathrm{wt} \% \mathrm{CTAB}$ and $0.15 \mathrm{wt} \%$ OP10.

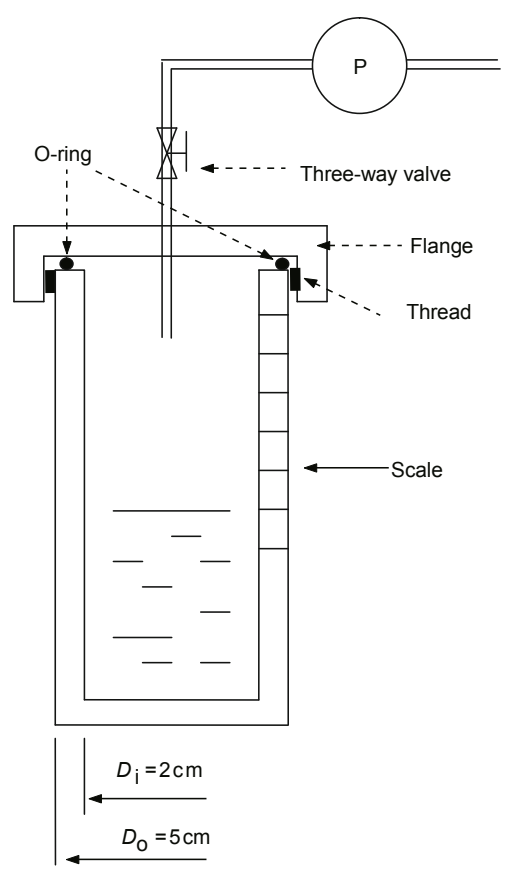

Fig. 1 Pressure-tight Plexiglas cylinder

\subsection{Experimental methods}

\subsubsection{Foam half-life}

The Ross-Miles test was used to produce foams and to compare their stability under atmospheric pressure. The halflife defined as the time required for the foam to collapse to half of its initial height, was used to characterize the stability of foams under atmospheric pressure.

However, when the initial pressure is higher than atmospheric pressure, the foam half-life measurements can not be made following Ross-Miles test because the glass used cannot tolerate a high pressure. The equipment, shown in Fig. 1 , was developed instead to test foam stability under aboveatmospheric pressure.

When the initial pressure was higher than atmospheric pressure, considering the size of the pressure-tight equipment used and the requirement of visual observation, foam was generated by chemical reaction instead of mechanical means due to its poor repeatability and stability. The experimental procedures for measuring foam half-life in the equipment we developed are described as follows:

(1) Prepare a $1.6 \% \mathrm{HCl}$ solution containing $0.15 \mathrm{wt} \%$ CTAB and $0.15 \mathrm{wt} \%$ OP- 10 , and add $20 \mathrm{ml}$ of such a solution into the equipment. Then place different amounts of $\mathrm{NH}_{4} \mathrm{NO}_{2}$ powder wrapped with a piece of plastic film above the solution in the graduated cylinder.

(2) Place a weight drop above the film and seal the equipment. Then shake the equipment to let the weight drop fall and make $\mathrm{NH}_{4} \mathrm{NO}_{2}$ mix with hydrochloric acid for reaction. Place the equipment in a thermostat water bath.

(3) Monitor the change of the foam height with time. When the height reached a maximum value, simultaneously record the pressures and bath temperatures. 


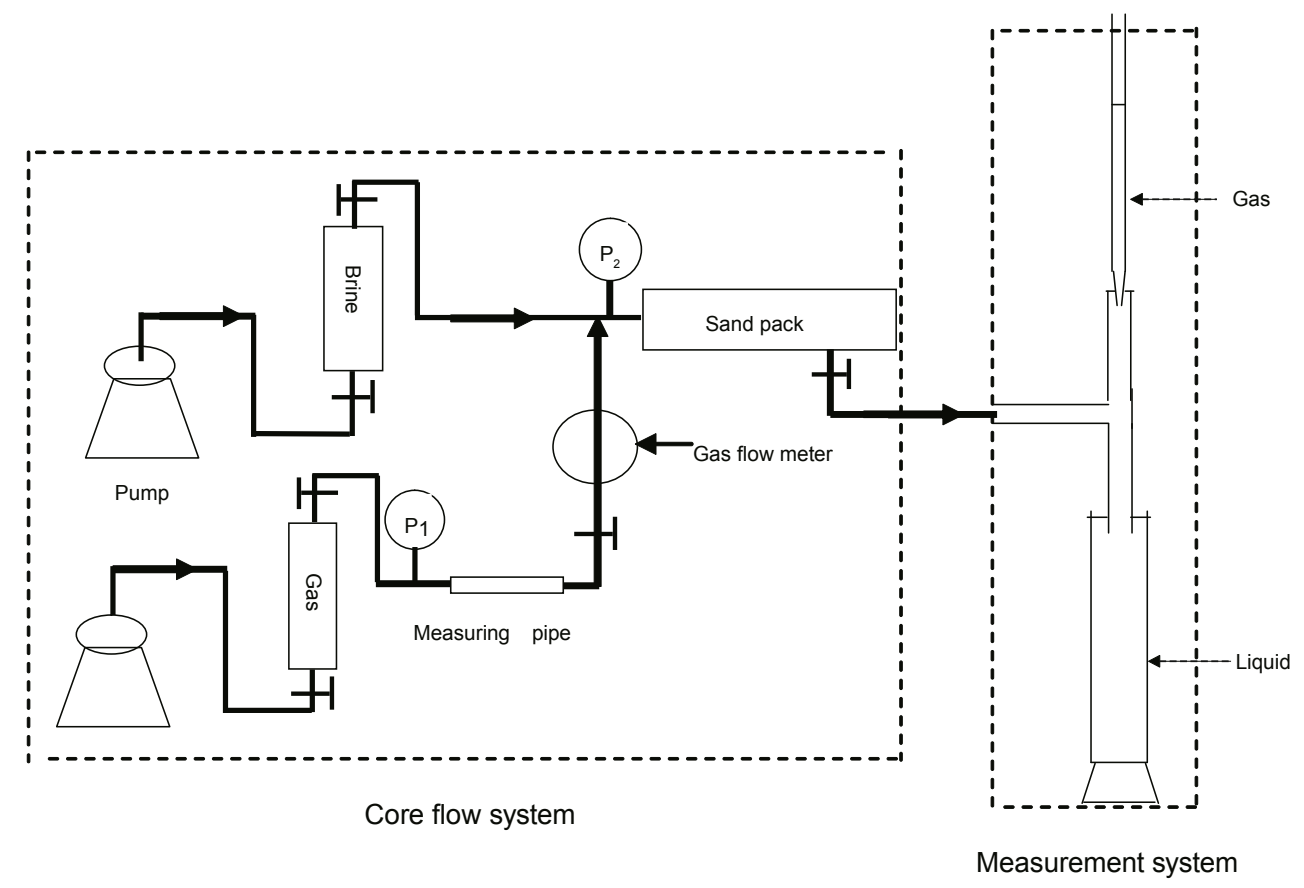

Fig. 2 Instrument for measuring stability of foam in sand pack models

The device was initially pressurized to a specific value with nitrogen. The pressure increased when the reaction proceeded, and reached a maximum value at the completion of reaction, and that value was taken as the initial pressure.

\subsubsection{Residual resistance factor}

When the gas and foaming solution are completely dispersed in porous media, foam stability changes with time. Breakdown of foams in porous media inevitably leads to decreases in the number of gas/liquid interfaces and the average capillary pressure in porous media, and in turn the flow resistivity of following gas or liquid. Therefore, the change of foam residual resistance factor to nitrogen or water with time can be used to characterize the stability or residence capability of gas after dispersion in porous media. A more stable foam will cause a slower decrease in residual resistance factor with time.

The residual resistance factor to nitrogen gas, which was caused by foam, was determined as follows:

(1) A sand pack (30 cm long, ID $2.8 \mathrm{~cm}$ ), filled with 100-160 mesh sand, was evaculated and then saturated with simulated formation water. Water was injected until the pressure was stabilized, then permeability and pore volume $(\mathrm{PV})$ were measured (PV is $50-51.2 \mathrm{ml}$, approximately 51 $\mathrm{ml})$.

(2) Nitrogen was injected at room temperature $\left(30^{\circ} \mathrm{C}\right)$ and a specified pressure drop $\Delta P_{1}(20-30 \mathrm{kPa})$, gas relative permeability $K_{0 \mathrm{~g}}$ was measured after the pressure was stabilized.

(3) $10.2 \mathrm{ml}(0.2 \mathrm{PV})$ foaming solution was injected at an injection rate of $5 \mathrm{ml} / \mathrm{min}$. Afterwards $24.26 \mathrm{ml}$ (standard conditions) nitrogen was injected at $\Delta P$, then the inlet and outlet of the sand pack were closed. The relative permeability to nitrogen gas $K_{1 \mathrm{~g}}$ was then measured at room temperature and $\Delta P_{1}(20-30 \mathrm{kPa})$ after the sand pack was placed in a 60
${ }^{\circ} \mathrm{C}$ thermostat for a specific period of time.

The sand pack was refilled, and the above procedures were repeated by only changing the $\Delta P$, then the change of residual resistance factor at different standing times was investigated.

The gas relative permeability was continually changing after foam was generated in porous media. Its value was calculated from injection pressure drop differential and gas flow rate at sand pack outlet. The gas relative permeability and residual resistance factor to gas could be expressed as follows:

Gas relative permeability

$$
K_{\mathrm{g}}=\frac{20 p_{\mathrm{sc}} Q_{\mathrm{sc}} \mu_{\mathrm{g}} L}{A\left(p_{1}^{2}-p_{2}^{2}\right)}
$$

Residual resistance factor to nitrogen gas

$$
R_{K}=\frac{K_{0 \mathrm{~g}}}{K_{\mathrm{lg}}}
$$

The water permeability values were measured before and after foaming when water injection rate was fixed at $5 \mathrm{ml} /$ $\min$. The water relative permeability and residual resistance factor to water were expressed as follows:

Water relative permeability

$$
K=\frac{10 Q \mu_{1} L}{A \Delta P}
$$

Residual resistance factor to water

$$
R_{K}=\frac{K_{01}}{K_{11}}
$$

where $p_{\mathrm{sc}}$ is atmospheric pressure, MPa; $p_{1}$ and $p_{2}$ are the inlet and outlet pressures of sand pack, MPa; $\Delta P$ is the pressure drop, MPa; $Q_{\text {sc }}$ is the gas volumetric flow rate at atmospheric 
pressure, $\mathrm{cm}^{3} / \mathrm{s} ; L$ is the length of the sand pack, $\mathrm{cm} ; \mu_{\mathrm{g}}$ and $\mu_{1}$ are viscosity of nitrogen and water at experimental temperature, $\mu_{\mathrm{g}}=0.0178 \mathrm{mPa} \cdot \mathrm{s}, \mu_{1}=0.8 \mathrm{mPa} \cdot \mathrm{s} ; K_{0 \mathrm{~g}}$ and $K_{\mathrm{lg}}$ are gas relative permeability, $\mu \mathrm{m}^{2} ; K_{01}$ and $K_{11}$ are water relative permeability, $\mu \mathrm{m}^{2}$.

\subsubsection{Initial residual resistance factor}

(1) Sand pack filled with outcrop sand, was evaculated and then saturated with simulated formation water. The influence of water injection rate on permeability was measured before the initial resistant test of foam was conducted.

(2) The same as Step (2) in Section 2.2.2.

(3) $24.26 \mathrm{ml}$ (standard conditions) of nitrogen was injected at fixed $\Delta P$ to initiate foaming, followed by 10.2 $\mathrm{ml}(0.2 \mathrm{PV})$ foaming agent solution with a rate of $5 \mathrm{ml} / \mathrm{min}$. Five minutes after foam production, gas relative permeability was measured at room temperature and injection pressure drop $\Delta P_{1}(20-30 \mathrm{kPa})$, then the residual resistance factor to nitrogen was calculated.

The sand pack was refilled and the above procedures were repeated, then the influence of an alternative injection pressure drop of gas on residual resistance factor were investigated. Experimental temperature was $30^{\circ} \mathrm{C}$.

\section{Influence of environmental pressure on foam stability}

\subsection{Foam stability in bulk phase}

Foam stability in bulk phase under elevated pressure was the same as that under atmospheric pressure. Foam height decreased with time as shown in Table 1. The higher the pressure, the slower the decrease of foam height, i.e., the longer the foam half-life and the higher the foam stability. Table 1 shows the influence of pressure and temperature on foam half-life.

Table 1 Variation of foam height with time at different initial pressures and temperatures

\begin{tabular}{|c|c|c|c|c|c|c|c|c|}
\hline \multirow{2}{*}{$\begin{array}{l}\text { Time } \\
\min \end{array}$} & \multicolumn{3}{|c|}{$\begin{array}{c}\text { Foam height at } 30^{\circ} \mathrm{C} \text { and under different initial } \\
\text { pressures, } \mathrm{cm}\end{array}$} & \multirow{2}{*}{$\begin{array}{l}\text { Time } \\
\min \end{array}$} & \multicolumn{4}{|c|}{$\begin{array}{l}\text { Foam height at } 60^{\circ} \mathrm{C} \text { and under different initial } \\
\text { pressures, cm }\end{array}$} \\
\hline & $0.1 \mathrm{MPa}$ & $0.305 \mathrm{MPa}$ & $0.65 \mathrm{MPa}$ & & $0.1 \mathrm{MPa}$ & $0.6 \mathrm{MPa}$ & $1.0 \mathrm{MPa}$ & 2.0 $\mathrm{MPa}$ \\
\hline 0 & 8.2 & 7.8 & 7.4 & 0 & 8.6 & 8.1 & 7.8 & 8.0 \\
\hline 20 & 7.7 & 7.7 & 6.8 & 20 & 6.2 & 6.4 & 6.6 & 6.9 \\
\hline 40 & 7.0 & 7.2 & 6.3 & 40 & 4.6 & 4.8 & 5.8 & 6.3 \\
\hline 80 & 5.7 & 6.2 & 5.7 & 60 & 3.4 & 4.3 & 5.3 & 5.8 \\
\hline 120 & 4.1 & 5.0 & 5.2 & 80 & & 3.6 & 4.7 & 5.3 \\
\hline 160 & 3.0 & 3.5 & 4.7 & 100 & & & 4.3 & 4.9 \\
\hline 200 & & & 4.3 & 120 & & & 3.9 & 4.5 \\
\hline 240 & & & 4.0 & 160 & & & & 4.3 \\
\hline 280 & & & 3.7 & & & & & \\
\hline
\end{tabular}

At a fixed temperature, foam half-life increased linearly with increasing environmental pressure (Fig. 3). The reason is that the pressure of gas inside the foam increases with increasing environmental pressure, gas phase density increases and the difference in density between liquid film and gas inside the foam decreases. Experiments were performed at a pressure of $0.1-2 \mathrm{MPa}$, and the pressure or density is statistically linear with the reciprocal of nitrogen volume in the range of 1-10 MPa. Consequently, the foam half-life can be determined by using the linear extrapolation method when the system pressure exceeds $2 \mathrm{MPa}$. According to the fitting result, foam half-life is prolonged to $800-900$ min when environmental pressure increases to $10 \mathrm{MPa}$. The foam half-life versus pressure curve at $60^{\circ} \mathrm{C}$ has a slope half of that at $30^{\circ} \mathrm{C}$, showing that a higher temperature leads to lower foam stability.

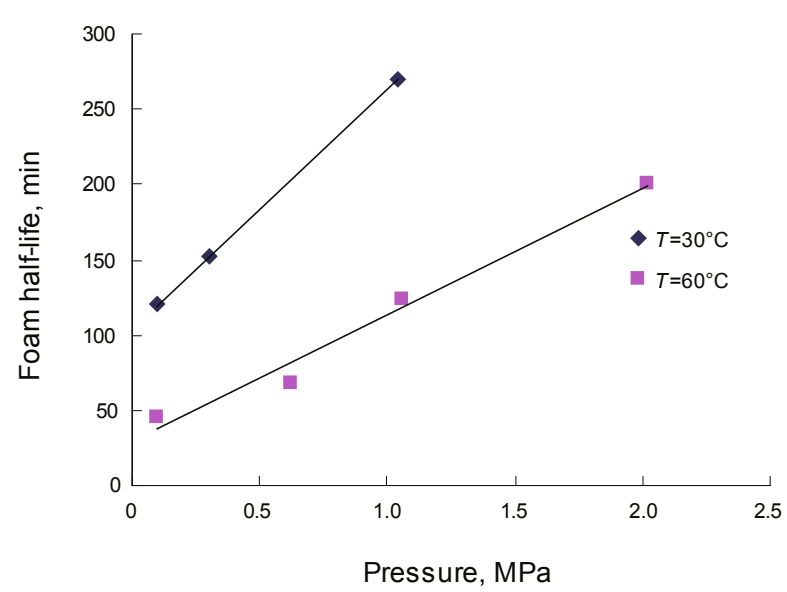

Fig. 3 Change of foam half-life with temperature and pressure 
It can be inferred that under reservoir conditions $(\geq 10$ $\mathrm{MPa}$ ) the foams, as the main gas/liquid dispersion system in porous media, will exist for a much longer time than observed under atmospheric pressure.

\subsection{Foam stability in porous media}

\subsubsection{Influence of injection pressure drop on foam dispersion}

Table 2 shows the effect of pressure drop on the permeability of the sand pack. The permeability of the sand pack varied in the range of $4.03-7.63 \mu \mathrm{m}^{2}$, and 3.73-4.98 $\mu \mathrm{m}^{2}$ in two tests, when the injected pressure drop was 0.031.0 MPa. Water injected into the sand pack would inevitably cause particle migration, resulting in change of permeability by 1-2 times. Under gas injection pressure drop of 0.02-0.8 $\mathrm{MPa}$, foams generated in the sand pack caused a change of relative permeability to follow-up gas or liquid by orders of magnitude. Due to its low density, the sand-carrying capability of gas was much smaller than water in the flow process, so the influence of gas flow rate on the migration of particles could be neglected. The change in permeability by orders of magnitude was caused by the increase in capillary pressure as a result of increased number of gas/liquid interfaces after foam generation, and in turn greater flow resistance and lower permeability. During determination of the permeability before and after foaming, the pressure drop should be kept stable to reduce the influence of injection rate on permeability.

Table 2 Variation of water permeability with pressure drop

\begin{tabular}{ccc}
\hline & \multicolumn{2}{c}{ Water permeability $K, \mu \mathrm{m}^{2}$} \\
Injection pressure drop & Test 1 & Test 2 \\
\cline { 2 - 3 } 0.03 & 4.03 & 3.73 \\
0.2 & 5.77 & 5.30 \\
0.3 & 5.28 & - \\
0.5 & 6.74 & 6.69 \\
0.8 & 5.16 & 4.98 \\
1.0 & 7.63 & - \\
\hline
\end{tabular}

Fig. 4 indicates that residual resistance factor to gas, $R_{\mathrm{k}}$, is an exponential function of gas injection pressure drop. With other conditions the same, the lower the permeability of sand pack, the greater the residual resistance factor. With increasing gas injection pressure drop, more gas molecules invade into micropores, resulting in an increase in the number of gas/liquid interfaces in the porous media and consequently a reduction in permeability in the sand pack and an increase in residual resistance factor.

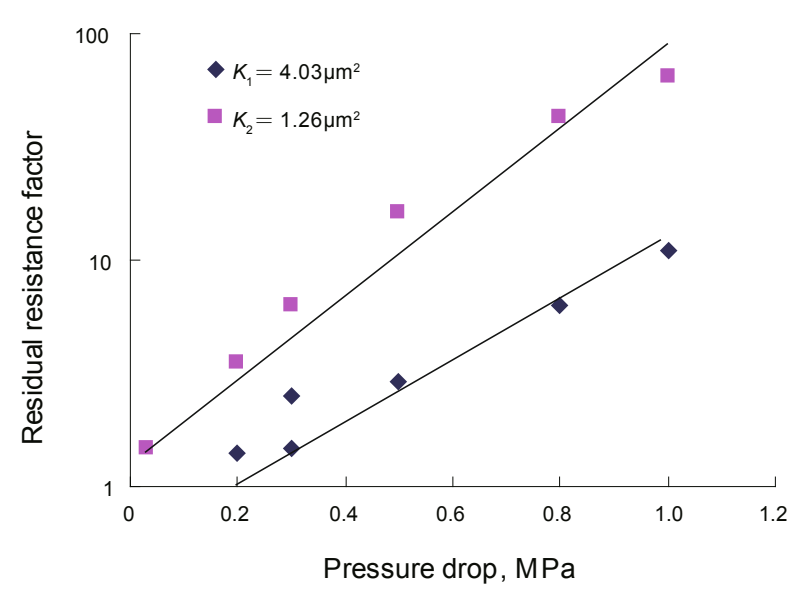

Fig. 4 Residual resistance factor versus pressure drop

\subsubsection{Foam stability in porous media}

Table 3 indicates the change of residual resistance factor to gas with time. At a longer time, the residual resistance factor of foam against nitrogen decreased and the residence capability of foam decreased. The residual resistance factor is an exponential function of time, as shown in Fig. 5.

Table 4 indicates the change of residual resistance factor to water with time. The residual resistance factor to water after foaming was 13.6 initially and slowly decreased to 7-8 after standing for 8 hours. From the curves in Fig. 5, residual resistance factor to water is an exponential function of time.

Table 3 Variation of residual resistance factor to gas with time

\begin{tabular}{cccc}
\hline $\begin{array}{c}\text { Time } \\
\mathrm{h}\end{array}$ & $\begin{array}{c}K_{0 \mathrm{~g}} \\
\mu \mathrm{m}^{2}\end{array}$ & $\begin{array}{c}K_{\mathrm{gg}} \\
\mu \mathrm{m}^{2}\end{array}$ & $K_{\mathrm{lg}} / K_{0 \mathrm{~g}}$ \\
\hline 1 & 6.82 & 0.87 & 7.84 \\
4 & 3.33 & 0.59 & 5.64 \\
8 & 2.25 & 0.42 & 5.36 \\
24 & 1.37 & 0.24 & 5.71 \\
\hline
\end{tabular}

Notes: $K_{0 \mathrm{~g}}-$ Gas permeability before foaming;

$K_{\mathrm{lg}}$-Gas permeability after foaming

Table 4 Residual resistance factor to water versus time

\begin{tabular}{|c|c|c|c|c|}
\hline \multirow{2}{*}{$\begin{array}{c}\text { Time } \\
\mathrm{h}\end{array}$} & \multirow{2}{*}{$\begin{array}{l}\Delta p_{0} \\
\mathrm{kPa}\end{array}$} & \multicolumn{2}{|c|}{$\Delta p_{1}, \mathrm{kPa}$} & \multirow{2}{*}{$\begin{array}{l}\text { Residual resistance } \\
\text { factor } K_{01} / K_{11}\end{array}$} \\
\hline & & Max. & Stabilized & \\
\hline 1 & 12.45 & 169.97 & 97.42 & 13.65 \\
\hline 4 & 25.48 & 246.3 & 246.3 & 9.66 \\
\hline 8 & 37.79 & 297.63 & 297.63 & 7.87 \\
\hline 24 & 62.10 & 438.24 & 354.38 & 7.05 \\
\hline
\end{tabular}

Notes: $\Delta p_{0}, K_{01}$-Pressure drop and water permeability before foaming; $\Delta p_{1}, K_{11}$-Pressure drop and water permeability after foaming 
Therefore, with increasing standing time, residual resistance factor to both nitrogen and water decreased, and the residence capability or stability of foam decreased. If residual resistance factor was taken as the expression of halflife in porous media, the time required for a decrease to one half was over 3 hours (for nitrogen) and even 8 hours (for water). While the half-life of foam in bulk phase was only 15-20 min and $25 \mathrm{~min}$ (stabilizer added). Therefore the static stability of foam in porous media was much better than that in bulk phase.

In addition, the initial residual resistance factor to water was considerable, but it decreased rapidly, because foam might break down on contacting with water. More importantly, with increasing water injected into the sand pack the foams trapped previously in porous media were gradually expelled, the number of gas/liquid interfaces decreased and capillary pressure then decreased. The initial residual resistance factor to gas was relatively small, but decreased slowly, which might be the cause of better blockage of gas by foam.

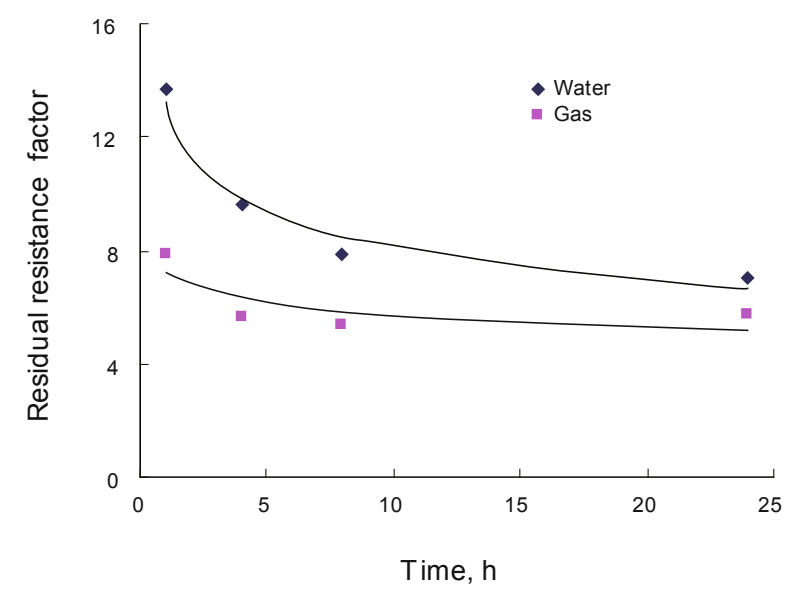

Fig. 5 Influence of standing time on foam residual resistance factor

\subsubsection{Mechanism analysis}

The necessary condition for gas to enter throat channels is that its initial capillary pressure should be greater than the capillary invasion pressure $P_{\mathrm{c}}^{\mathrm{e}}$. For a pore throat with round cross section, $P_{\mathrm{c}}^{\mathrm{e}}$ can be calculated with the following equation:

$$
P_{\mathrm{c}}^{\mathrm{e}}=\frac{2 \sigma}{R_{\mathrm{t}}}
$$

where $\sigma$ is gas/liquid surface tension; and $R_{\mathrm{t}}$ is throat channel radius.

Once gas dispersion is completed, its stability depends on the pressure differential between pores and throat length (Fig. 6). The greater the ratio of pore radius to throat radius, the greater the pressure differential between two bubbles in the pore, the weaker the foam stability, and consequently the shorter the residence time of foam in porous media.

The greater the throat length and the thicker the liquid film between bubbles, the higher the foam stability.

Therefore, stability and residual resistance factor of foam not only are related to permeability, but also are determined by the pore structure (including throat channel length and ratio of pore radius to throat radius).

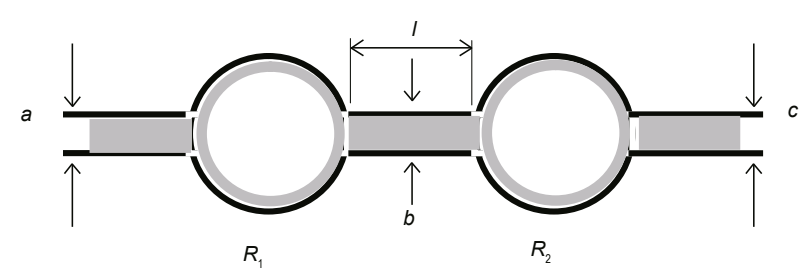

Fig. 6 Distribution of gas/liquid in pores and throats (Dark color is pore and rock wall, white color is gas, gray color is water)

The greater the gas flow rate in the pore and throat, the lower the surface tension, the better the dispersion, and consequently the greater the resistance to the flow of followup fluids.

\section{Field applications}

\subsection{Field overview}

Block Xiaowa is a heavy oil reservoir in Liaohe Oilfield, Bohai Bay Basin, China, with an average porosity of 0.29 and permeability of .5-1.0 $\mu^{2}$. W35527, W3839, W3241 and Waguan-10 are four steam-stimulated wells producing from $\mathrm{D}_{3}$ interval about 1,240-1,430 $\mathrm{m}$ in depth. After multiple steam stimulation processes, a large amount of water condensed from injected steam accumulated at the $\mathrm{D}_{3}$ bottom. The condensed water would absorb most of the heat carried by following injected steam and reduce the effective heating area, leading to high water cut and poor steam stimulation response. Actually, the four wells had seen a 98\% water cut at the end of the fifth cycle of steam injection. To improve their steam stimulation response, it was decided to inject $\mathrm{N}_{2}$ into W35527, W3839 and W3241 and to inject $\mathrm{N}_{2}$ foam in Waguan-10 in February, 2007.

\subsection{Injection process}

The injection rate was set at $700 \mathrm{~m}^{3} / \mathrm{h}$. When the injection pressure reached $20 \mathrm{MPa}$, the injection process was stopped, then the well was shut-in for seven days. The final injection volumes of $\mathrm{N}_{2}$ into W35527, W3839 and W3241 were about $6 \times 10^{4} \mathrm{~m}^{3}$, and the volume into Waguan-10 was $5 \times 10^{4} \mathrm{~m}^{3}$ accompanied by 5 tons of foaming agent.

\subsection{Results and discussion}

When re-opened for production, the three $\mathrm{N}_{2}$ treated wells reached the production rate peaks about 2 to 20 days later, and came back to the $98 \%$ water cut about 7 to 30 days later. That is to say, the maximum effective life of $\mathrm{N}_{2}$ treatment was 30 days.

In contrast, the production rate reached its peak about 30 days later in Waguan-10 and then declined obviously more slowly than those of the other three wells. During a 349-day production period, the average daily oil production increased from $0.2 \mathrm{t}$ to $3.34 \mathrm{t}$ by a daily increase of $3.1 \mathrm{t}$ and 
the cumulative oil incremental production was $1,162 \mathrm{t}$. As a matter of fact, the $\mathrm{N}_{2}$ foam treatment was still effective in June, 2008 and its effect would continue.

There are two main reasons for the observation that $\mathrm{N}_{2}$ foam treatment works more effectively than $\mathrm{N}_{2}$ treatment. One reason is that the foam chamber is located above condensed water due to its lower density and the steam subsequently injected can preferentially heat the oil-rich zone in the high position of oil formation (Fig. 7). And the other one is that the residence of foam in the formation reduces the decline rate of residual resistance factor, thus decreasing the production rates of water and $\mathrm{N}_{2}$.

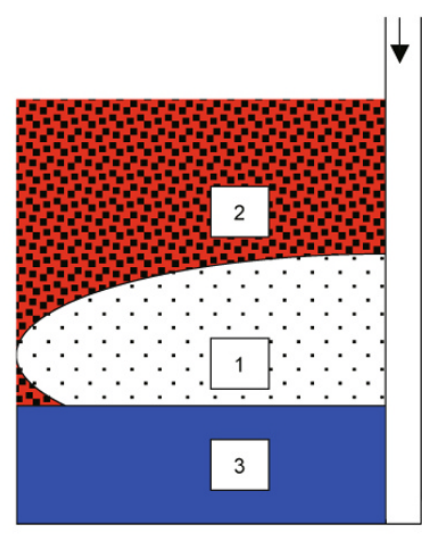

(a) Gas injection

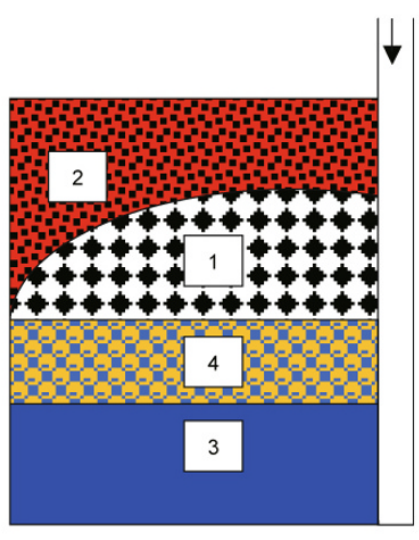

(b) Foaming+gas injection
1. Steam heating zone, 2. Oil-rich zone, 3. Condensed water, 4. Foam barrier

Fig. 7 Influence of nitrogen foam on steam heating and water control

\section{Conclusions}

1) Foam stability was investigated in a pressure-tight Plexiglas equipment and sand pack. Foam stability in bulk phase (expressed as half-life) increased linearly with increasing environmental pressure, while foam dispersion and stability in porous media (expressed as residual resistance factor) was much greater than the stability in bulk phase.

2) Foam dispersion was related to the gas flow rate in porous media. With the same permeability, in the experimental flow rate range the greater the flow rate (or the invasion pressure), the better the gas dispersion, and the greater the residual resistance factor of foam. At a specific gas injection pressure drop, the lower the sand pack permeability, the greater the residual resistance factor.

3) Foam stability in porous media depends on the pore structure of porous media, including pore/throat radius ratio, throat channel length and heterogeneity, as well as formation permeability and gas flow rate.

4) $\mathrm{N}_{2}$ foam presents different behavior from $\mathrm{N}_{2}$ in porous media. Its longer residence time in formation and higher flow resistivity allow $\mathrm{N}_{2}$ foam to control water and gas production rates, thus delaying formation energy depletion and enhancing treatment effects. The mechanisms can explain the field observation that $\mathrm{N}_{2}$ foam works more effectively than $\mathrm{N}_{2}$.

\section{References}

Barigou M, Deshpande N S and Wiggers F N. An enhanced electrical resistance technique for foam drainage measurement. Colloids and Surfaces A: Physicochemical and Engineering Aspects. 2001. 189(1/3): 237-246

Bergeron V, Fagan M E and Radke C J. Generalized entering coefficients: a criterion for foam stability against oil in porous media. Langmuir. 1993. 9(7): 1704-1713

Bernard G G and Holm L W. Effect of foam on permeability of porous media to gas. Soc. Petrol. Eng. J. 1964. 4(32): 267-274

Bond D C and Holbrook O C. Gas Drive Oil Recovery Process. US Patent 2866507. 1958

Cohen R, Exerowa D, Kolarov T, et al. Foam films stabilized with lysophosphatidylcholine: a comparison of microinterferometric and fourier transform infrared spectroscopy thickness measurements. Langmuir. 1997. 13(12): 3172-3176

Deshpande N S and Barigou M. Foam flow phenomena in sudden expansions and contractions. International Journal of Multiphase Flow. 2001. 27(8): 1463-1477

Greaves M. Air injection-improved oil recovery strategy for the UK continental shelf. The Oil \& Gas Review. 2004. 118-121

Holm, L W. Mechanism of gas and liquid flow through porous media in the presence of foam. SPE Journal. 1968. 8(4): 359-369 (SPE 1848)

Iglesias E, Anderez J, Forgiarini A, et al. A new method to estimate the stability of short-life foams. Colloids and surfaces A: Physicochemical and Engineering Aspects. 1995. 98(1-2): 167-174

Jiang L, Zhao F, Tang J A, et al. Experimental study of oil-water interface dilatation rheological properties. Chinese Science Bulletin. 2000. 45(23): 2501-2505 (in Chinese)

Kose K. 3D NMR imaging of foam structures. Journal of Magnetic Resonance, Series A. 1996. 118(2): 195-201

Kovscek A R, Patzek T W and Radke C J. Mechanistic foam flow simulation in heterogeneous and multidimensional porous media. SPE Journal. 1997. 2(4): 511-526 (SPE 39102)

Kovscek A R, Tang G Q and Radke C J. Verification of Roof snap off as a foam-generation mechanism in porous media at steady state. Colloids and Surfaces A: Physicochem. Eng. Aspects. 2007. 302(1-3): 251-260

Li Z M, Sun M S, Lin R Y, et al. Laboratory study on foam plugging and selective divided-flow. Acta Petrolei Sinica. 2007. 28(4): 115-118 (in Chinese)

Ma B Q. A study on liquid hold-up of foam. Journal of Xi'an Shiyou University (Natural Science Edition). 1994. 9(4): 48-51 (in Chinese)

Müller H J and Rheinländer Th. Anomalous thickness variation of nonionic surfactant foam films with salt concentration. Langmuir. 1996. 12(10): 2334-2339

Raza S H. Foam in porous media: characteristics and potential applications. Soc. Pet. Eng. J. 1970. 328-336

Rossen W R. Theory of mobilization pressure gradient of flowing foams in porous media. II. Effect of compressibility. Journal of Colloid and Interface Science. 1990. 136(1): 17-37

Rossen W R. Snap-off in constricted tubes and porous media. Colloids and Surfaces A: Physicochemical and Engineering Aspects. 2000. 166(1-3): 101-107

Zhu T, Ogbe D O and Khataniar S. Improving the foam performance for mobility control and improved sweep efficiency in gas flooding. Ind. Eng. Chem. Res. 2004. 43(15): 4413-4421

(Edited by Sun Yanhua) 